\title{
GESTIÓN DEL AGUA CON ENFOQUE PARTICIPATIVO EN GUATEMALA FRENTE AL CAMBIO CLIMÁTICO
}

\section{WATER MANAGEMENT WITH A PARTICIPATORY APPROACH IN GUATEMALA IN THE FACE OF CLIMATE CHANGE}

Referencia del artículo

Ochoa, W. (2020). Gestión del agua con enfoque participativo en Guatemala frente al cambio climático. Revista Científica del SEP. 3(1), 33-37. DOI: https://doi.org/10.36958/sep.v3i01.30

\author{
Wener Armando Ochoa Orozco \\ wenerochoa@hotmail.com \\ Ingeniero Agrónomo, MSc. Gestión Ambiental \\ Universidad de San Carlos de Guatemala
}

Fecha de recibido: $11 / 06 / 2020$

Fecha de aceptado: 18/10/2020

\begin{abstract}
RESUMEN
Guatemala es un país con una ubicación geo estratégica, ya que está en la zona de convergencia inter tropical, el cual es un factor que contribuye a la diversidad biológica y a la significativa riqueza hídrica con la que cuenta. Asimismo, cuenta con una riqueza cultural extraordinaria.

A pesar de ello el $45 \%$ de la población del país carece de agua y saneamiento, sumado a ello, está ubicado en el séptimo lugar a nivel mundial en materia de riesgo de desastres, con altos niveles de deforestación y afectada seriamente por fenómenos hidrometeorológicos asociados al cambio climático como inundaciones, sequías, huracanes, tormentas, depresiones tropicales, así como incremento poblacional, políticas públicas poco eficientes de gestión, corrupción, falta de financiamiento y de tecnología moderna, etc. Ante esta problemática la gestión del agua con enfoque participativo se convierte en una alternativa urgente y de sostenibilidad para reducir los conflictos ya existentes a causa de este vital liquido y evitar futuras tensiones.
\end{abstract}

El presente artículo describe, analiza y explica los riesgos de la falta de agua para el país, para luego plantear lineamientos orientadores para la gestión del agua con enfoque participativo y género para adaptarnos al cambio climático.

\section{PALABRAS CLAVE}

Gestión del agua, escases de agua, cambio climático, participación, género

\section{ABSTRACT}

Guatemala is a country with a geo-strategic location, since it is in the inter-tropical convergence zone, which is a factor that contributes to biological diversity and the significant water wealth it has. It also has an extraordinary cultural wealth.

Despite this, $45 \%$ of the country's population lacks water and sanitation, in addition to this, it is located in seventh place worldwide in terms of disaster risk, with high levels of deforestation and seriously affected by phenomena hydrometeorological associated with climate change such as floods, droughts, hurricanes, storms, tropical depressions, as well as population growth, inefficient public management policies, corruption, lack of financing and modern technology, etc. Faced with this problem, water management with a participatory approach becomes an urgent and sustainable alternative to reduce existing conflicts due to this vital liquid and avoid future tensions.

This article describes, analyzes and explains the risks of the lack of water for the country, to then propose guiding guidelines for water management with a participatory and gender approach to adapt to climate change.

\section{KEYWORDS}

Water management, water scarcity, climate change, participation, gender 


\section{INTRODUCCIÓN}

Guatemala cuenta con una extensión territorial de 108,889 $\mathrm{km} 2$, una población de 18.2 millones de habitantes, distribuidas territorialmente en 22 departamentos, 340 municipios y tres regiones hidrológicas o vertientes (Pacífico, Atlántico y Golfo de México), 46 cuencas, 100,599 millones metros cúbicos de agua superficial y 33,699 millones de metros cúbicos de agua subterránea, dando un total de 134,298 millones de metros cúbicos de agua en total.

Sin embargo, el $60 \%$ de las aguas superficiales están contaminadas y aproximadamente el $5 \%$ de las municipalidades cuentan con sistema de tratamiento de aguas residuales. Debido al cambio de uso de la tierra y la poca planificación territorial es evidente el deterioro de los bienes naturales ocasionados por la erosión de suelos, poco conocimiento de la contaminación de acuíferos, manejo inadecuado de los desechos sólidos, crecimiento urbano desordenado, etc. (Instituto Nacional de Estadística INE, Ministerio de Ambiente y Recursos Naturales MARN, Secretaria General de Planificación SEGEPLAN, Instituto de Sismología, Vulcanología, Meteorología e Hidrología INSIVUMEH).

La gestión del agua se entiende como aquel proceso que busca dar soluciones de carácter técnico científico, social y político al aprovechamiento eficiente y sostenible del bien hídrico a través del desarrollo y gestionar de forma coordinada el agua, la tierra y los bienes conexos, tratando de maximizar el bienestar social y económico, de una manera equitativa y sin comprometer el nivel de sostenibilidad de los ecosistemas (Global Water Partnership GWP, 2019).

Es en este contexto que la gestión del agua tanto superficial como subterránea juega un papel determinante para el país ya que hasta ahora se tiene pocos estudios y muy pocas experiencias de participación pública en la gestión del bien hídrico.

Sin embargo, no se tiene experiencia de gestión participativa del agua en Guatemala, a pesar de tener una estructura con potencial para ello, como lo es el Sistema de Consejos de Desarrollo y poca visibilidad de acciones en el marco de cumplimiento de los objetivos del desarrollo sostenible, principalmente el número 6 Agua limpia y saneamiento; 3 Salud y Bienestar, 13 Acción por el Clima, los comités comunitarios pro mejoramiento de agua, alcaldías indígenas, entre otros, que tienen participación e influencia desde un nivel comunitario hasta nacional. Ante ello, se hace cada vez mas importante implementar la gestión participativa de los bienes hídricos con enfoque de género y multicultural, ante la escasez de agua que se registra en los últimos años en diversas partes del país, así como conocer sus características físico químicas, hidrodinámicas, geodésicas y geológicas, para que este bien sea sustentable para las generaciones presentes y futuras.

Cabe resaltar que en cuanto a la perspectiva de género; el acceso de agua para las familias es básico, sin embargo, a nivel rural la carga laboral de recolección del vital líquido es para la mujer, las niñas y niños, exponiéndose en muchos de los casos a un clima de inseguridad por las distancias que recorren para la colección de agua.

En el contexto actual de la pandemia de COVID-19, esta propuesta es estratégica para la aplicación de las medidas de prevención en la utilización del vital líquido, para reducir la vulnerabilidad de este derecho humano a nivel comunitario en donde escasamente tienen cosechadores de agua de lluvia o riachuelos no aptos para el consumo humano.

\section{METOdOLOGÍA}

El artículo tiene como premisa que el cambio climático afecta directamente el bien hídrico y es necesario emprender hacia una gestión participativa del mismo.

Para ello, se llevó a cabo una metodología mixta: cualitativa y cuantitativa. En la fase de investigación cualitativa se identificaron actores claves, y se realizó una entrevista semi estructurada con el propósito de obtener información sobre la temática investigada y tener un registro de las percepciones que existen entre los actores claves del tema.

En la fase de investigación cuantitativa se revisaron bases de datos, información hidroclimática proporcionada por el INSIVUMEH, informes científicos y datos proporcionados por diversas instituciones oficiales y académicas, para generar una matriz comprensible para analizar los datos, las interrelaciones que tienen y obtener conclusiones.

Ambos enfoques, conllevaron una revisión bibliográfica extensa que abarcó artículos científicos, informes de organismos internacionales, documentos de trabajos, artículos académicos y estudios referidos al tema realizados por diversas instituciones.

El estudio es descriptivo, dado su carácter y hace uso del método analítico socio-crítico, específicamente la metodología descriptiva y explicativa. Esquemáticamente el abordaje del tema se hizo en dos momentos, una primera etapa se refirió a la conceptualización de la temática y la segunda etapa abordó el análisis y la propuesta de lineamientos generales para la gestión del agua con enfoque participativo en Guatemala frente al cambio climático. 
ema se hizo en dos momentos, una primera etapa se refirió a la conceptualización de la temática y la segunda etapa abordó el análisis y la propuesta de lineamientos generales para la gestión del agua con enfoque participativo en Guatemala frente al cambio climático.

Fundamento legal de la participación:

Ley de Desarrollo Social: Decreto 42-2001

Ley de Consejos de Desarrollo Urbano y Rural:

Decreto 11 - 2002

- $\quad$ Ley General de Descentralización: Decreto Número 14-2002

- Código Municipal: Decreto Número 12-2002

Instrumentos de planificación:

Política general de Gobierno

Planes de desarrollo municipales

Planes de desarrollo departamentales

Planes de desarrollo regional

Política Nacional de Desarrollo K’atún 2032

Objetivos de desarrollo sostenible

Otras Políticas sectoriales relacionadas

Estructuras de participación:

Consejos Comunitarios de Desarrollo de I y II nivel.

Asociaciones de vecinos.

Alcaldías comunitarias.

Alcaldías indígenas.

Consejo Municipal de Desarrollo.

Comités Únicos de Barrio.

Consejo Departamental de Desarrollo.

Consejo Regional de Desarrollo.

Según Fundación para la Conservación del Agua FUNCAGUA (2019), hay municipios de Guatemala que manifiestan descenso en los niveles piezométricos dinámicos y estáticos parciales de agua subterránea, asimismo, en informes científicos se pronostica aumento de temperatura de 0.5 a 3.5 grados centígrados para los próximos 30 años y una reducción de precipitación para el país hasta del 30\% para finales de siglo, impactando mas a los departamentos de Baja Verapaz, Sacatepéquez, Totonicapán, Chimaltenango, Guatemala, El Progreso, Zacapa, Jutiapa, Chiquimula y el sur de Quiché y Huehuetenango ( Rivera, et al., 2019).

El aumento de la temperatura media anual y la disminución de la precipitación que se prevén para el año 2030, debido a los efectos del cambio climático, tendrán impactos significativos en la agricultura de todo el país. También es probable que las áreas aptas para los cultivos que sustentan la seguridad alimentaria campesina, cambien en el futuro. Algunos municipios ganarán aptitud productiva para ciertos cultivos pero que se debe de analizar siempre en el marco de la sustentabilidad y considerando los impactos ambientales; y otros la perderán en especial por la escasez de agua (Ochoa, 2018).

\section{ANÁLISIS Y DISCUSIÓN}

Ante el crecimiento exponencial de la población y el avance de la urbanización, así como la escasez y contaminación del agua superficial, el agua subterránea, sin duda alguna es una fuente de provisión de agua por medio de pozos y redes de distribución en diferentes partes del país. Por ello es necesario comenzar a lograr la gestión participativa de este bien, mediante la caracterización actualizada de las aguas superficiales y subterráneas para luego analizar su calidad física, químicas, bacteriológica, hidráulica y otras características en función de las actividades socioeconómicas alrededor.

De acuerdo con Rivera, et al. (2019), se proyectan sequías más prolongadas y alteraciones en la temporada de lluvias, que generarían un mayor número de tormentas locales severas y esto afectará seriamente las áreas de recarga hídrica, así como el abastecimiento a la población en general. Para McKean (2000), las aguas subterráneas son bienes que comparten virtudes tanto de carácter público como privado. Es acá donde se valora la gestión participativa de los bienes hidrogeológicos ya que sin importar si es público o privado, el uso de este bien por una persona puede afectar la disponibilidad para otros potenciales usuarios; es ante esta realidad que se plantea gestión del agua con enfoque participativo para evitar el agotamiento y avanzar hacia una gestión sostenible de dicho bien y para ello se propone hacer uso del sistema de consejos de desarrollo y de las estructuras organizativas locales existentes.

Implementar la estrategia de pueblos, etnias y comunidades afrodescendientes contenida en la Estrategia Regional de Cambio Climático (ERCC) de la Comisión Centroamericana de Ambiente y Desarrollo (CCAD) y el Sistema de la Integración Centroamericana (SICA) formulada en noviembre de 2010, la cual busca fortalecer e implementar los sistemas, conocimientos y prácticas tradicionales indígenas para la adaptación y mitigación del cambio climático en los planes, programas y proyectos sobre dicha materia (Banco Mundial, 2019).

En aras de encontrar soluciones a la gestión del agua, se considera que la participación de todos los sectores es fundamental. Sin embargo, cabe aclarar que es posible encontrar limitantes en algunos Consejos de Desarrollo durante su implementación dada la misma naturaleza y complejidad del agua, lo que requerirá gestionar mecanismos adecuado, pertinentes y contextualizados. Por ahora, es el mecanismo legal con que se cuenta en la actualidad; dado que ha habido desde hace algunos años planteamientos de reformas a ley 
por varios factores, entre ellas lo permisiva que es en el plano político; que en el fondo el tema del agua tiene una arista política también.

Desde la perspectiva de la GWP, la gestión integrada del agua debe ser un proceso que comprometa tanto a las autoridades internacionales, gubernamentales, locales, al sector privadocomo a la sociedad entera, con el objetivo de evitar confrontaciones y prevenir desequilibrios en los ecosistemas. El enfoque de la GWP destaca no sólo una visión participativa sino también un compromiso en los modelos educativos de la gestión y utilización del agua (Nieto, 2011).

Ante los impactos del cambio climático y la carencia de una leyde aguas en el país y la poca coordinación inter institucional alrededor del agua, hace imperante atender la gestión integradadel bien hídrico y aprovechar de manera eficiente y sostenible del agua. Esto con el fin de adaptarnos al cambio climático y mejorar la calidad del agua para consumo humano, así como atender el saneamiento ambiental donde el tratamiento y procesamiento de las aguas residuales de manera urgente.

\section{CONCLUSIONES}

Se le debe dar un abordaje inter y transdisciplinario al agua en el país, haciendo uso de la alianza del sector público con las instituciones académicas y organizaciones no gubernamentales y sociales como alcaldías indígenas, COCODE, comités únicos de bario, comités comunitarios pro mejoramiento de agua, así como con el sector privado en los territorios donde sea factible. Todo este proceso rumbo a la construcción y validación de la ley de aguas que regule dicho bien en el país.

Se debe iniciar por gestionar el agua en diversos niveles, comenzando desde el comunitario a través de los Consejos Comunitarios de Desarrollo -COCODE- hasta donde sea posible según los cursos de agua superficial y subterránea, luego atender el nivel municipal haciendo uso de los Consejos Municipales de Desarrollo -COMUDE, y de acuerdo a las caracterizaciones hidrológicas e hidrogeológicas obtenidas gestionar el agua a nivel departamental y regional a través delos Consejo Departamentales de Desarrollo -CODEDE- y de los Consejos Regionales de Desarrollo.

Las municipalidades tienen la responsabilidad básica de la prestación de los servicios de agua potable y saneamiento. Sin embargo, existe una diversidad de instituciones gubernamentales y no gubernamentales que también han asumido este rol por lo que se deben coordinar a nivel municipal haciendo uso del COMUDE.
Sistematizar otras experiencias innovadores de gestión del agua, como, por ejemplo, en el área rural del municipio de Cobán, Alta Verapaz las comunidades se organizan para colectar fondos y contratan a una empresa internacional quienes vienen a perforarles su pozo de hasta 400 a 500 pies de profundidad, y de esta manera obtener acceso al agua y lavisión es tener agua entubada en sus viviendas.

La calidad y cantidad del bien hídrico disponible es cada día menor, y se maneja en forma sectorial desde el punto de vista institucional.

La multiplicidad, duplicidad y sobre posición de diferentes leyes e instituciones impiden la protección y una adecuada y eficiente gestión del agua.

\section{AGRADECIMIENTOS}

Este artículo fue elaborado en el marco del curso de Participación Comunitaria y Género en Proyectos de Agua, el cual es parte del pensum de la Maestría en Gestión de Recursos Hidrogeológicos, de la Facultad de Ingeniería de la Universidad de San Carlos de Guatemala, coordinada por el Centro de Estudios Superiores de Energía y Minas CESEM- y apoyada por el proyecto Agua Futura, de la cooperación italiana.

Asimismo, agradezco las revisiones y aportes de los siguientesprofesionales:

Lic. En Antropología Fredman Pacay

Lic. En Ciencias Jurídicas y Sociales Vilma Luna

Ing. En Recursos Naturales Renovables Bayron González

\section{REFERENCIAS BIBLIOGRÁFICAS}

Banco Mundial. (2019). Informe Hacia una Centroamérica mas resiliente. Pilares para la acción. GrupoBanco Mundial.

Cuerpo de Ingenieros de los Estados Unidos de América. (2000). Evaluación de Recursos de Agua de Guatemala. Guatemala.

FUNCAGUA. (2019). Análisis piezométrico de pozos de agua para los municipios de la Mancomunidad Gran Ciudad del Sur: Amatitlán, Mixco, San Miguel Petapa, Santa Catarina Pínula, Villa canales y Villa Nueva, Guatemala. Fundación para la Conservación del Agua en la Región Metropolitana de Guatemala (FUNCAGUA). Guatemala. 
Global Water Partnership GWP. (2019). Hoja de Datos sobre el Estado de la Gestión Hídrica en Guatemala 2017-2018. Guatemala.

Instituto de Agricultura, Recursos Naturales y Ambiente IARNA-. (2002). El Agua: Situación Actual y necesidades de gestión. Guatemala: Universidad Rafael Landívar.

Instituto Nacional de Estadística (2019). XII Censo Nacional de Población y VII de Vivienda 1985-2019, Recuperado de: http: / / redatam.censopoblacion.gt/bingtm/RpWebEngine - exe/ Portal?BASE=CPVGT2018\&lang=esp. Visitada 05/05/2020.

Ministerio de Ambiente y Recursos Naturales Renovables MARN. (2016). Informe Ambiental del Estado Guatemala. Guatemala.

McKean, M. (2000). Common Property: What it is, what is it good for, and what makes it work. In: People and forests: Communities, institutions and governance. Gibson, C.C., Mckean, M.A., and E. Ostrom, eds. MIT Press, Cambridge, Massachusets. Pp: 27-55.

Ministerio de Salud Pública y Asistencia Social. (2013). Política Nacional del Sector de Agua Potable y Saneamiento. Guatemala.

Nieto, N. (2011). La gestión del agua: tensiones globales y latinoamericanas. Política y cultura, (36), 157-176. Recuperado en 18 de mayo de 2020, de http: / / www.scielo.org.mx/scielo.php?script=sci_arttext\& pid=S0188-77422011000200007\&lng=es\&t lng=es.

ONU. (1992). Convención marco de las Naciones Unidassobre el Cambio Climático. Nueva York.

Ochoa, W., Rivera, P., Salguero, M., y González, B. (2016).Uso de la tierra y variabilidad climática en la subcuenca del río Panajachel, cuenca del lago de Atitlán, Sololá, Guatemala. XII Conferencia Científica Internacional. Universidad de Ciego de Ávila Máximo Gómez Báez. ISBN 978-959-16-3165-7
Ochoa, W., Morales, L., Vivar, V., y Rodríguez, M. (2018). Impacto del cambio climático en la seguridad $y$ soberanía alimentaria en Guatemala. Revista Análisis de la Realidad Nacional. Universidad de San Carlos de Guatemala. ISSN 2227- 9113

Rivera, P., Bardales, W., y Ochoa, W. (2019). Escenarios futuros de cambio climático para Guatemala. Primer reporte de evaluación del conocimiento sobre cambio climático en Guatemala. (pp. 40-61). Guatemala: Editorial Universitaria UVG.

SEGEPLAN. (2006). Política nacional de gestión integradade los recursos hídricos (PNGIRH). Guatemala. p.33.(2006a). Estrategia para la gestión integrada de los recursos hídricos de Guatemala. Guatemala. p. 83.

Sistema Guatemalteco de Ciencias del Cambio Climático SGCCC. (2017). Primer reporte de evaluación del conocimiento del cambio climático en Guatemala. Guatemala: Sistema Guatemalteco de Ciencias del Cambio Climático. Recuperado el 3 de 5 de 2020, de http://sgccc.org.gt/reporte-de-cambio- climaticoguatemala/informacion-general-del-primer-reporte- deevaluacion-del-conocimiento-del-cambio-climatico-enguatemala/

\section{Sobre autor}

\section{Wener Armando Ochoa Orozco}

Es Profesor de Posgrados de Ingeniería y Agronomía de la Universidad de San Carlos de Guatemala, USAC, Ingeniero Agrónomo, Maestría en Gestión Ambiental y Doctorado en cambio climático y sostenibilidad por la Universidad de San Carlos de Guatemala, Posgrados en Meteorología por el Centro Nacional de Investigación Atmosférica UCAR, Gestión de riesgos agrarios y ambientales de la Universidad Politécnica de Madrid, España, Agua y Sustentabilidad por la Universidad de Córdoba, Argentina, y de Hidrogeología por el Centro Universitario del Norte de la USAC, Conferencista nacional e internacional en temas de Ambienta, Agua, Cambio climático y Desarrollo sostenible, Miembro del Sistema Guatemalteco de Ciencias del Cambio Climático de Guatemala.

Copyright (c) 2020 Wener Armando Ochoa Orozco

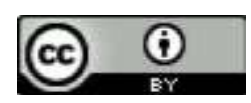

Este texto está protegido por una licencia Creative Commons 4.0.

Usted es libre para Compartir - copiar y redistribuir el material en cualquier medio o formato - y Adaptar eldocumento - remezclar, transformar y crear a partir del material- para cualquier propósito, incluso comercialmente, siempre que cumpla la condición de:

Atribución: Usted debe reconocer el crédito de una obra de manera adecuada, proporcionar un enlace a la licencia,e indicar si se han realizado cambios. Puede hacerlo en cualquier forma razonable, pero no de forma tal que sugieraque tiene el apoyo del licenciante o lo recibe por el uso que hace.

$\underline{\text { Resumen de licencia }}$ - Texto completo de la licencia 